\title{
OPTIQUE ATTOSECONDE ET ÉLECTRONIQUE PÉTAHERTZ DANS LES SEMICONDUCTEURS
}

\author{
Hamed Merdfi ${ }^{\mathbf{*}}$, Willem Boutu \\ ${ }^{1}$ Université Paris-Saclay, CEA, CNRS, LIDYL, 91191 Gif-sur-Yvette, France \\ *hamed.merdji@cea.fr
}

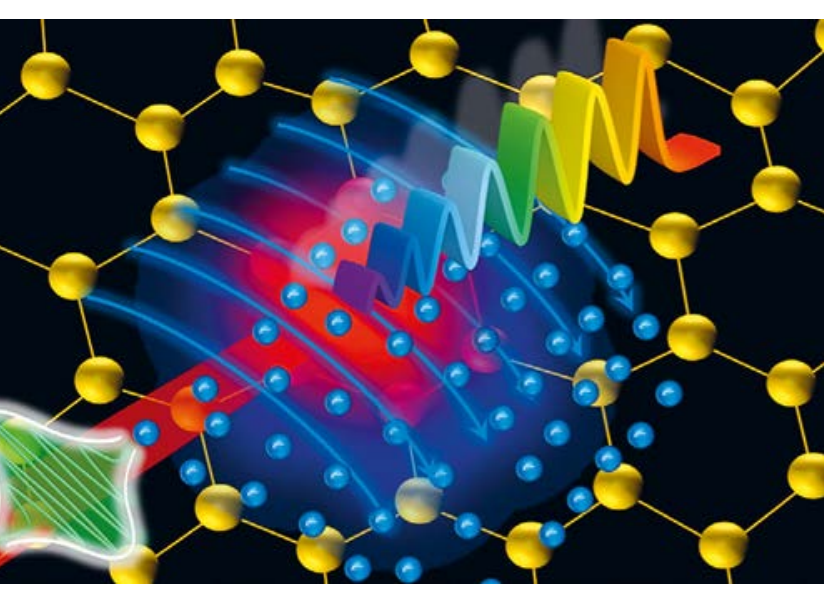

\begin{abstract}
La génération d'harmoniques laser d'ordre élevé dans les cristaux semiconducteurs est une nouvelle source de rayonnement ultra-bref (femtoseconde à attoseconde) cohérent et de courte longueur d'onde (de l'ordre de quelques dizaines de nanomètres) à très haute cadence. Outre la versatilité de cette source pour les applications, offerte notamment par les progrès en nano-structuration, l'étude du rayonnement émis permet de suivre les dynamiques électroniques ultrarapides au sein de matériaux complexes.
\end{abstract}

Article publié en accès libre sous les conditions définies par la licence Creative Commons Attribution License CC-BY (https://creativecommons.org/licenses/by/4.0), qui autorise sans restrictions l'utilisation, la diffusion, et la reproduction sur quelque support que ce soit, sous réserve de citation correcte de la publication originale.

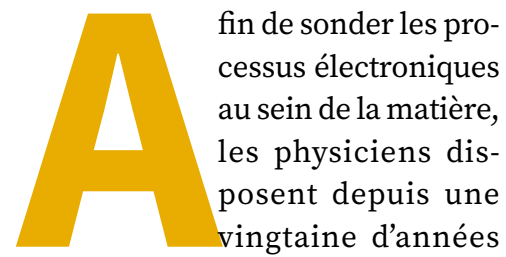

d'un nouvel outil, le laser attoseconde. L'attoseconde, c'est-à-dire le milliardième de milliardième de seconde ( 1 as $=10^{-18} \mathrm{~s}$ ), représente l'échelle de temps caractéristique des dynamiques électroniques suite à une excitation par une impulsion lumineuse ou lors des premières étapes de réarrangement électronique lors des réactions chimiques. Cependant, de telles échelles de temps sont inaccessibles par les impulsions laser standards, dont les durées d'impulsions sont limitées par la durée du cycle optique de la lumière visible à au mieux quelques femtosecondes. Un phénomène mis en évidence au CEA Saclay à la fin des années 1980, la génération d'harmoniques d'ordre élevé (GHOE), a permis l'essor des lasers attosecondes [1]. L'interaction non linéaire entre une impulsion laser infrarouge, femtoseconde et intense et un milieu gazeux (généralement constitué de gaz rare) peut résulter en l'émission d'un train d'impulsions attosecondes dans le domaine de l'extrême ultraviolet (EUV). Depuis, il a été démontré que l'émission de ce rayonnement pouvait être limitée à une impulsion attoseconde unique, dont la durée record est aujourd'hui de l'ordre de 40 as. Les impulsions attosecondes ont permis ces dernières années des avancées spectaculaires dans de nombreux domaines, tels que l'observation en temps réel de la photoionisation d'un atome d'hélium à proximité d'une résonance, la mesure de migrations de charges dans des molécules biologiques ou l'observation en temps réel de l'ionisation d'électrons au sein d'un matériau. Cependant, cette technologie est encore limitée à quelques laboratoires spécialisés dans le monde. Elle nécessite en effet l'accès à des lasers femtosecondes intenses de 
a)
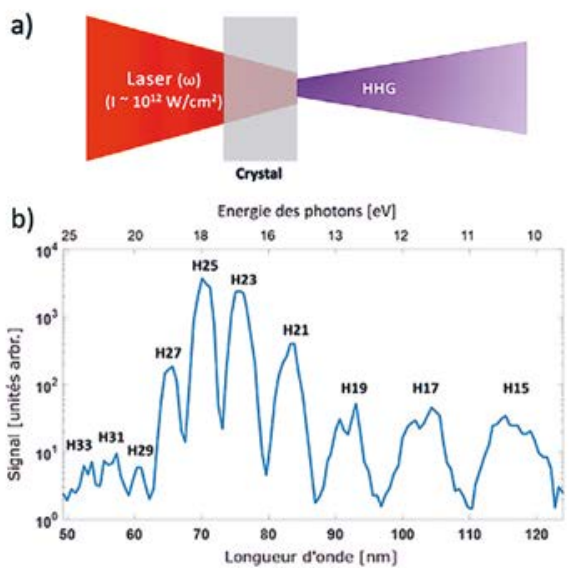

pointe (délivrant des énergies par impulsions de l'ordre du millijoule ou sub-millijoule) et des environnements ultra contrôlés. Néanmoins, depuis une dizaine d'années une alternative prometteuse a pris son essor. En 2011, un groupe de recherche de l'Université de Stanford a démontré un phénomène similaire à la GHOE suite à l'interaction entre des impulsions laser et un cristal semiconducteur d'oxyde de zinc (ZnO). Depuis cette première observation, ce type de rayonnement a été observé par l'équipe d'Hamed Merdji dans de nombreux matériaux, des cristaux semiconducteurs aux matériaux bidimensionnels tels que le graphène [3]. Bien que les énergies de c)

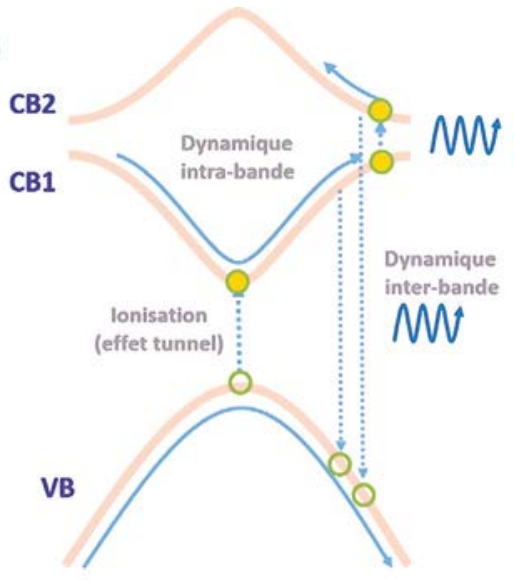

Figure 1. a) Schéma de principe de la génération d'harmonique d'ordre élevé dans les cristaux. Un faisceau laser femtoseconde est focalisé en face arrière d'un cristal, résultant en l'émission de photons de plus courtes longueurs d'onde. Un spectre typique est représenté en b) (résultant de l'interaction entre un laser de longueur d'onde centrale $\lambda=1.8$ $\mu \mathrm{m}$ dans un cristal de MgO). c) Dynamique électronique schématisé dans l'espace réciproque (CB : bande de conduction, VB: bande de valence).

photons atteintes ne soient pas encore aussi élevées que dans le cas des gaz, les harmoniques d'ordre plus élevé atteignent plusieurs dizaines d'electron-volts. Les lasers utilisés pour la GHOE dans les cristaux sont de tailles beaucoup plus modestes, le phénomène ayant été observé même avec des impulsions de moins de 10 nanojoules. Son efficacité est néanmoins suffisamment efficace pour permettre des premières applications.

\section{ORIGINE ÉLECTRONIQUE DE L'ÉMISSION HARMONIQUE}

Du fait des valeurs des champs électriques mis en jeu, la GHOE dans les cristaux se situe dans un régime très différent du régime dit perturbatif, commun en optique non linéaire « traditionnelle ». Au contraire, le régime est celui dit du champ fort, pour lequel le champ électrique du laser est du même ordre que le potentiel des atomes et qui privilégie une ionisation par effet tunnel. Bien que la réalité soit plus complexe, une vision simplifiée permet de se représenter les mécanismes électroniques mis en œuvre. Ce modèle en trois étapes est similaire à celui développé avec succès pour expliciter le phénomène dans les gaz. Lorsque le champ électrique atteint son maximum, un électron peut être ionisé par effet tunnel de la bande de valence à une bande de conduction (voir Fig. 1). Cet

\section{- SPECTROGON}

Filtres Interférentiels

De 200 á 15000 nm

- Passe-bande

- Passe-haut

- Passe-bas

- Large bande

- Densité neutre

- Disponible en stock

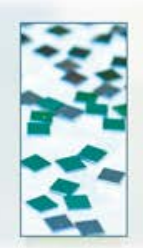

UK (parle francais): sales.uk@spectrogon.com·Tel +44 1592770000

Sweden (headquarters): sales se@spectrogon com $\cdot$ Tel +4686382800

US: sales.us@spectrogon.com·Tel+19733311191 
a)

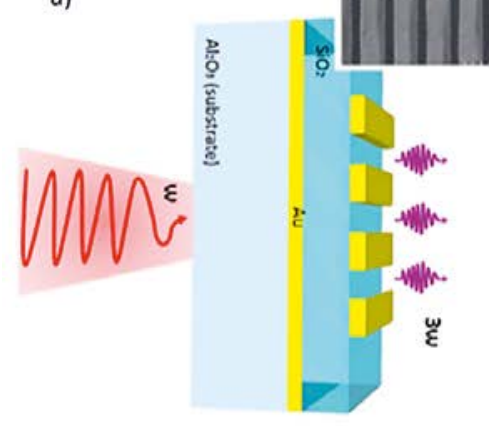

b)

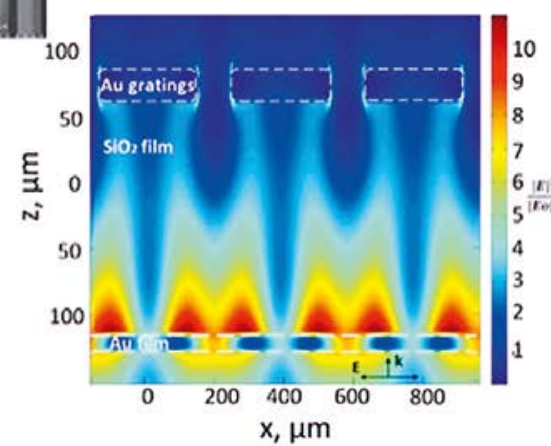

c)

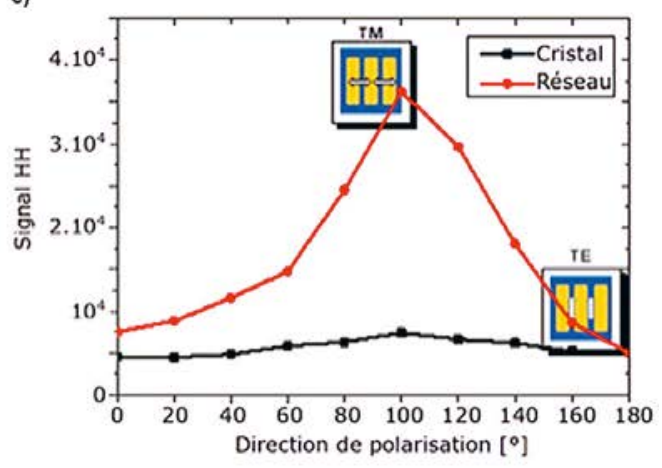

Figure 2. Exemple de génération d'harmoniques d’ordre élevé assistée par effet plasmonique. a) Schéma de principe, avec en insert une image électronique du réseau d'or. b) Simulation de l'intensité du champ électrique du laser au sein de la structure résonnante. On observe une augmentation locale d'un facteur 10, qui résulte en une augmentation du signal de plus d'un ordre de grandeur (c). Lorsque la direction de la polarisation du laser est tournée de $90^{\circ}$, la structure n'est plus résonnante et l'effet disparait.

électron va ensuite osciller au sein de cette bande, oscillations résultant en l'émission de rayonnement. Il peut aussi se recombiner avec un trou dans la bande de valence, en libérant l'énergie gagnée sous forme de rayonnement de courte longueur d'onde. La répétition de ce phénomène à chaque demi-cycle optique du laser incident résulte dans le domaine spectral en un spectre discret, constitué des ordres harmoniques impairs de la fréquence fondamentale. Cette image simpliste, provenant directement de l'approximation de l'électron actif unique utilisée en physique atomique, permet néanmoins d'obtenir une explication réaliste à certains comportements observés dans les cristaux. La réponse harmonique est ainsi directement liée à la réponse au champ laser des électrons dans le diagramme de bandes du cristal. Cette remarque a de multiples implications, tant sur le plan pratique que fondamental. En effet, il est ainsi possible de contrôler finement les caractéristiques de lémission en contrôlant la géométrie et les symétries de l'interaction. Un exemple immédiat consiste à tourner la direction de polarisation linéaire du champ laser par rapport à l'axe de symétrie du cristal à l'échelle du cycle optique en vue de confiner l'émission temporellement et d'émettre une impulsion attoseconde unique. Audelà, il est aussi possible d'utiliser une polarisation elliptique ou circulaire, voire même variable au cours de l'impulsion, ce qui permet de contrôler aussi le profil temporel de l'émission à l'échelle attoseconde. Inversement, a)

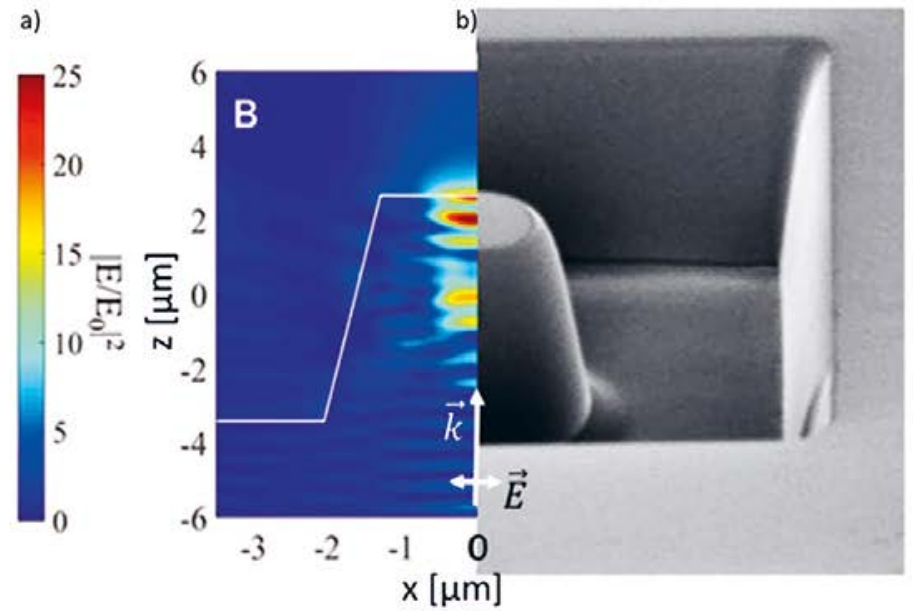

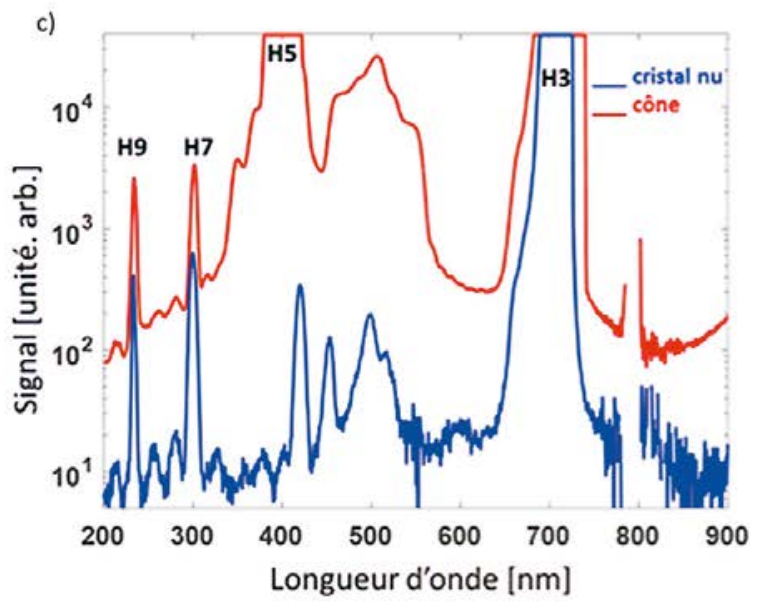

Figure 3. Exemple de génération d'harmoniques d'ordre élevé assistée par confinement. a) Simulation de l'intensité du champ électrique du laser au sein de la structure conique. On observe une augmentation locale d'un facteur 25. b) Image électronique d'un nanocône en ZnO. Le cône, identique à la simulation a), apparaît plus petit du fait de l'angle d'observation. c) Spectre harmonique mesuré dans le cristal de $\mathrm{ZnO}$ sans gravure (courbe bleue) et pour un laser couplé ( $\lambda=2,1 \mu \mathrm{m}, 20 \mathrm{MHz}$ ) avec le nanocône (courbe rouge). Suivant les conditions expérimentales, le signal harmonique peut être augmenté de plus de deux ordres de grandeurs. 
caractériser le rayonnement émis, pour un ensemble d'éclairements laser et d'orientations relatives, devrait permettre de reconstruire la structure de bandes du cristal. En outre, la caractérisation temporelle attoseconde de l'émission harmonique pourrait permettre l'étude de phénomènes transitoires ultrarapides, tels que des transitions isolant-métal ou la renormalisation de la bande interdite lors de l'interaction laser-matière. Enfin, l'émission harmonique est, nous l'avons dit, directement liée aux oscillations d'un courant d'électrons créé par l'impulsion laser à des fréquences Petahertz. La technologie laser permet depuis peu de parfaitement contrôler le profil temporel du champ laser, à une échelle plus petite que le cycle optique. En utilisant des impulsions et des matériaux ad-hoc, il devient en principe ainsi possible de contrôler finement ces courants électroniques, aux temps attosecondes. L'émission harmonique permettra de caractériser ces courants à l'échelle attoseconde. Ce principe est actuellement au centre de projets visant à développer de nouveaux dispositifs opto-électroniques commutant à des fréquences Petahertz, plusieurs ordres de grandeur plus rapidement que les dispositifs électroniques actuels (www.petacom.fr). Cependant, la mesure du profil temporel de l'émission harmonique n'a pas encore été démontrée. Elle repose en effet sur des mesures de phase spectrale développées pour la science attoseconde dans les gaz, qu'il s'agit de transposer sur un rayonnement dont le flux est moins important et l'énergie de photons moins élevée.

\section{POTENTIEL DES LASERS EUV DANS LES CRISTAUX POUR LES APPLICATIONS}

Le développement de la génération d'harmoniques d'ordre élevé dans les cristaux est tributaire des développements récents de la technologie laser, et plus particulièrement des lasers femtosecondes à forte puissance moyenne dans le proche et le moyen infrarouge. En effet, ce domaine de longueur d'onde permet d'augmenter le seuil de dommage des matériaux, plus aptes par conséquent à supporter les très forts éclairements nécessaires (avec des champs électriques de lordre du V/Å). Les taux de répétition très élevés (de $100 \mathrm{kHz}$ à plusieurs $\mathrm{MHz}$ ) ouvrent la voie aux utilisateurs de la communauté synchrotron. L'utilisation d'un matériau solide dans la GHOE présente de nombreux avantages par rapport à son pendant dans les gaz. Le premier, le plus évident, repose sur la simplicité du dispositif et sa compacité. Les éclairements nécessaires sont de plusieurs ordres de grandeur inférieurs. Par conséquent, il n'est plus nécessaire d'utiliser des lasers amplifiés reposant sur la technologie d'amplification par dérive de fréquence. Malgré des progrès continus, ces systèmes sont généralement complexes, et leurs taux de répétition sont limités à quelques kHz. De plus, il est possible de tirer profit des dernières avancées en micro-structuration des matériaux. Ainsi, des structures plasmoniques résonnantes peuvent être utilisées pour augmenter localement le champ laser, et augmenter l'émission harmonique globale d'un à deux ordres de grandeurs (Fig. 2, [4]). Ces structures métalliques sont cependant très fragiles, et leurs durées de vie dans le champ laser intense est parfois limitée à quelques secondes au mieux. Une solution alternative consiste à travailler directement le cristal, en y gravant par exemple des micro-guides d'onde coniques qui, en concentrant spatialement le champ laser en leur sommet permet de gagner localement un ordre de grandeur en éclairement (figure 3)[5]. Au-delà d'une simple augmentation de l'efficacité de conversion, il est possible de manipuler directement les propriétés spatio-temporelles du rayonnement harmonique en gravant sur la face arrière du cristal des

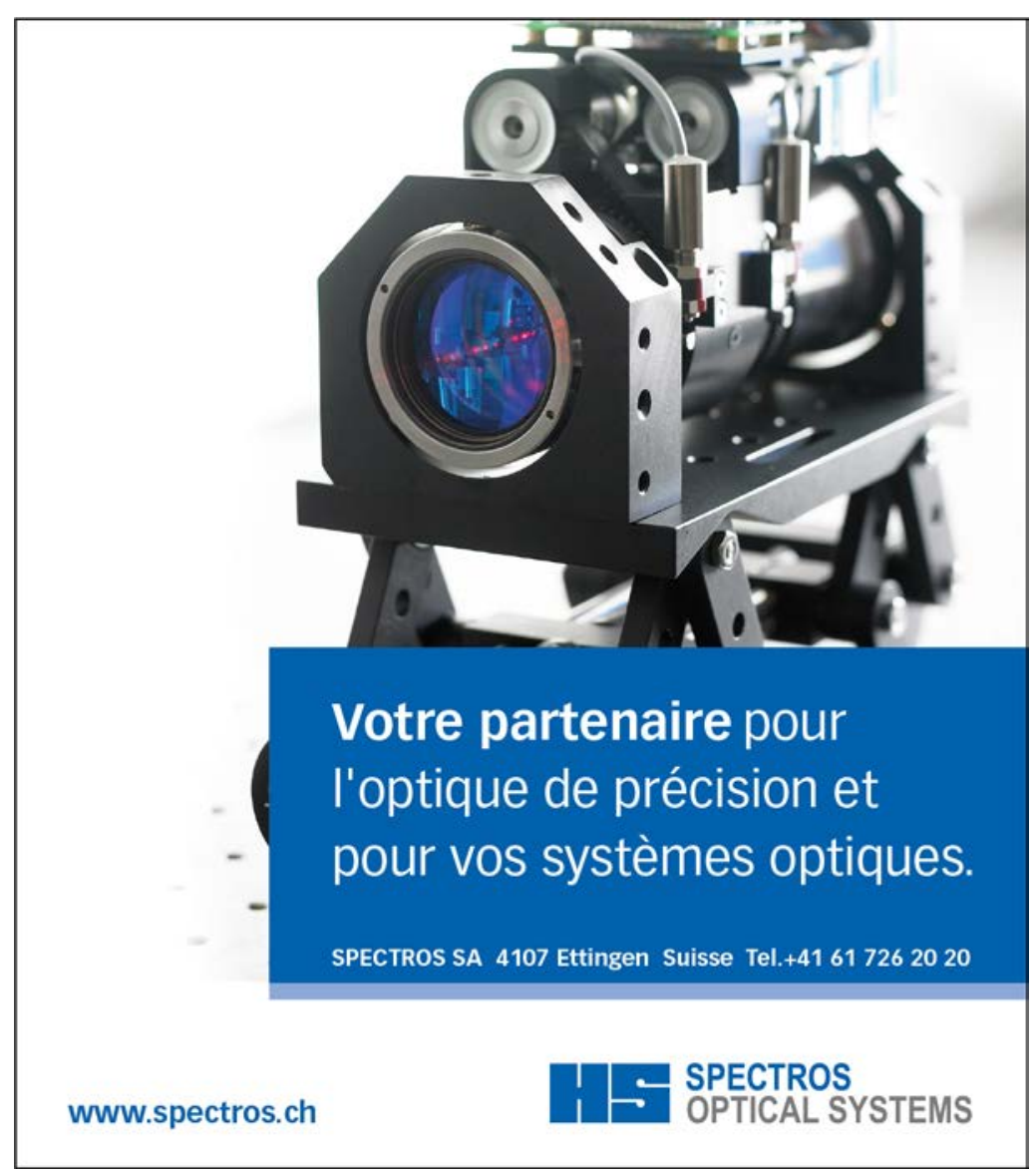


a)

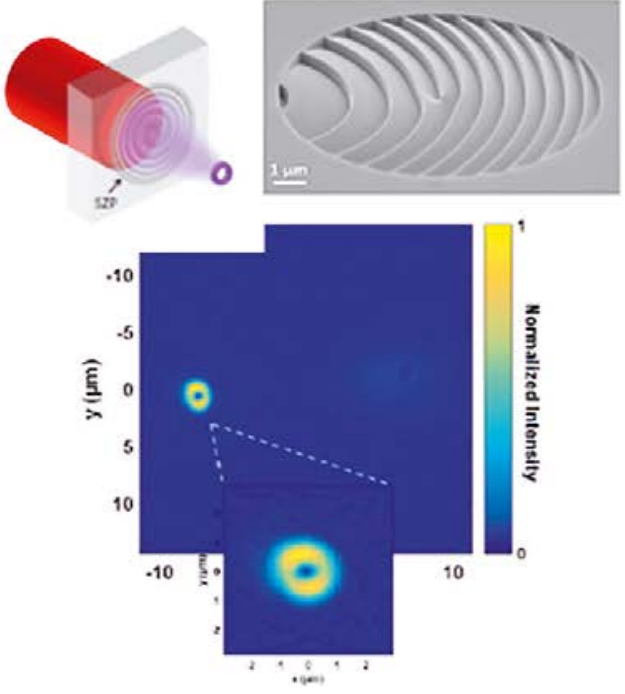

b)

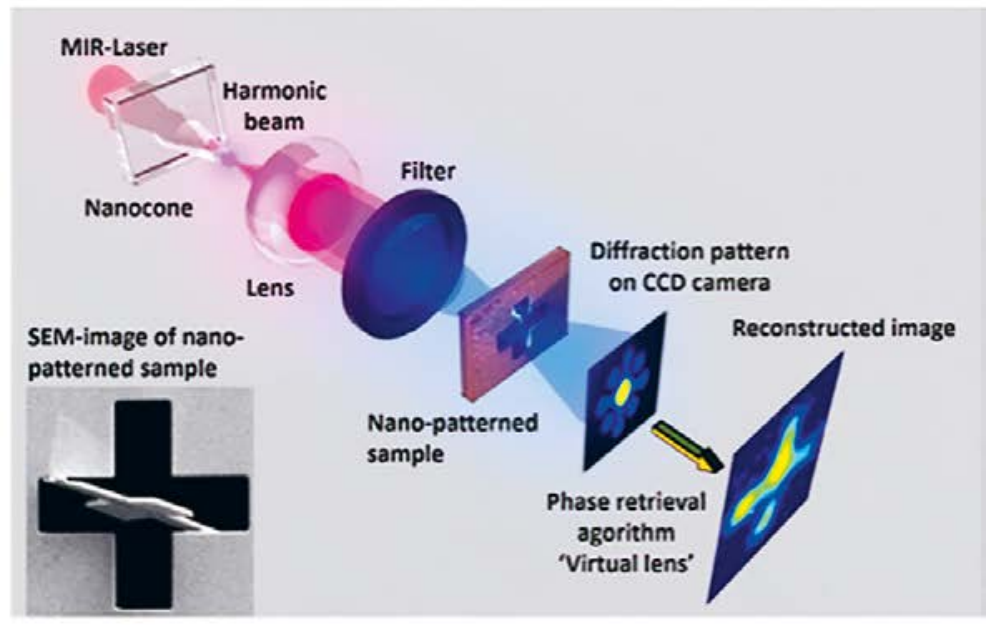

Figure 4. a) Exemple de manipulation des propriétés spatio-temporelles du faisceau harmonique. Une structure, ici une zone de Fresnel hors-axe présentant une fourche en son centre permet de focaliser l'harmonique désirée (ici, l'ordre 5 d'un laser à 2,1 $\mu \mathrm{m}$ ) tout en introduisant un moment angulaire orbital. b) Exemple d'application d'un laser harmonique solide à l'imagerie sans lentille. En insert, une image électronique de l'échantillon, une croix transparente gravée par faisceau d'ions focalisé au sein d'une membrane opaque, est visible (la barre blanche mesure $2 \mu \mathrm{m}$ ).

structures appropriées par faisceau d'ions focalisés. La figure 4a illustre l'utilisation de zones de Fresnel avec une singularité en leur centre pour à la fois focaliser l'ordre harmonique souhaité et lui donner par exemple un moment angulaire orbital arbitraire, visible ici par la structure annulaire du profil d'intensité du faisceau au foyer [6].

Bien que l'efficacité de la GHOE soit relativement faible (de l'ordre de $10^{-6}$ à $10^{-8}$ pour les ordres 9 et plus), le flux de photons est suffisant pour les applications, et ce notamment grâce au taux de répétition élevé. La figure $4 \mathrm{~b}$ illustre une expérience de microscopie sans lentille [5], une technique d'imagerie bien maitrisée par l'équipe d'Hamed Merdji [7]. Le faisceau UV émis par un nanocône est filtré (ici, l'ordre 5 d'un laser à $2,1 \mu \mathrm{m}$, soit $420 \mathrm{~nm}$ ) puis focalisé sur l'échantillon. Une caméra CCD enregistre l'intensité du champ diffracté en champ lointain. Un algorithme itératif, faisant office de « lentille virtuelle », reconstruit l'image de l'échantillon. Ces techniques d'imagerie sans lentille sont typiquement utilisées sur synchrotron dans le domaine des rayons $\mathrm{X}$, pour lequel il est difficile de fabriquer des optiques de reprise d'image efficaces et peu aberrantes.

\section{CONCLUSION}

La génération d'harmoniques d'ordre élevé dans les cristaux semiconducteurs offre de nouvelles perspectives aux sources attosecondes de laboratoire. Compactes et à très haut taux de répétition, faciles à manipuler grâce aux progrès de la nano-fabrication,

\section{RÉFÉRENCES}

[2] S. Ghimire and D. A. Reis, Nat. Phys. 15, 10 (2019)

[4] R. Nicolas, L. Shi, B. Chanteau et al., Plasmonics (2021)

https://doi.org/10.1007\%2Fs11468-021-01444-3

[5] D. Franz et al., Sci. Rep. 9, 5663 (2019)

[6] D. Gauthier et al., Opt. Lett. 44, 546 (2019)

[7] J. Huijts et al., Nat. Photonics 14, 618 (2020) elles se posent comme une alternative crédible aux sources actuellement disponibles. En dopant les semiconducteurs, nous avons observé très récemment que l'émission harmonique XUV pouvait être boostée. Les longueurs d'onde actuellement disponibles, limitées au VUV (140-100 nm) et au proche EUV (100-50 nm), sont néanmoins très intéressantes pour la physique du solide, la femtochimie et la spectroscopie de molécules biologiques. En outre, cette approche offre un nouvel outil pour étudier les dynamiques électroniques ultrarapides au sein de la matière condensée lors des interactions entre laser et matière. Plus récemment, nous avons démontré que la GHOE, processus de champ fort, est accompagnée de l'émission électrons cohérents de taille nanométrique et dont la structure attoseconde doit encore être élucidée [5].

[1] P. Salières et al., Quand les lasers deviennent atto, Photoniques 48, 40 (2010)

[3] Thèse de Dominik Franz 2028 \& Shatha Kaassamani, (2020), Paris Saclay 